\title{
Momentum Transfer from Quantum Vacuum to Magnetoelectric Matter
}

\author{
B. A. van Tiggelen, ${ }^{1}$ G. L. J. A. Rikken, ${ }^{2}$ and V. Krstic ${ }^{2}$ \\ ${ }^{1}$ Laboratoire de Physique et Modélisation des Milieux Condensés, Maison des Magistères, \\ CNRS/UJF BP 166, 38042 Grenoble, France \\ ${ }^{2}$ Laboratoire National des Champs Magnétiques Pulsés, CNRS/INSA/UPS, BP 14245, \\ 143 Avenue de Rangueil, 31432 Toulouse, France \\ (Received 26 September 2005; published 6 April 2006)
}

\begin{abstract}
A recent publication [Phys. Rev. Lett. 92, 020404 (2004)] raises the possibility of momentum transfer from zero-point quantum fluctuations to matter, controlled by applied electric and magnetic fields. We present a Lorentz-invariant description using field-theoretical regularization techniques. We find no momentum transfer for homogeneous media, but predict a very small transfer for a Casimir-type geometry.
\end{abstract}

DOI: 10.1103/PhysRevLett.96.130402

PACS numbers: 03.50.De, 42.50.Nn, 42.50.Vk

The Feigel hypothesis [1] (FH hereafter) suggests a nonzero total momentum density of zero-point fluctuations [2] in magneto-electric (ME) matter. ME effects can occur in media associated with a few specific symmetry classes [3], but can be induced in any medium, including the real quantum vacuum [4], by an external electric field $\mathbf{E}_{0}$ and magnetic field $\mathbf{B}_{0}$. The $\mathrm{FH}$, based on the manifestation of $\mathrm{ME}$ in optical birefringence, is controversial [5-7], yet important since it raises the possibility to transfer momentum from zero-point fluctuations to matter, controlled in direction and magnitude by the externally applied fields.

In ME media, photons with wave vectors $\mathbf{k}$ and $-\mathbf{k}$ behave differently, though independent of their polarization. Optical ME effects have been observed in birefringence [8-10] and in absorption [11-13]. The final result of Ref. [1] is an expression for the momentum density $\rho \mathbf{v}$ (mass density $\rho$, velocity $\mathbf{v}$ ) of a homogeneous medium in crossed, uniform, stationary fields $\mathbf{E}_{0}$ and $\mathbf{B}_{0}$ :

$$
\rho \mathbf{v}=\frac{\hbar}{32 \pi^{3} c_{0}^{4}}\left(\mu^{-1}+\varepsilon\right) \omega_{c}^{4} \chi \mathbf{E}_{0} \times \mathbf{B}_{0} .
$$

Here $\mu$ and $\varepsilon$ are the usual optical constants, $\chi$ is the ME coupling parameter, and $\omega_{c}$ is a cutoff frequency. The first problem is the Lorentz variance of Eq. (1). All quantities in the second factor of Eq. (1) transform under a Lorentz transformation, and do not leave Eq. (1) invariant. The second problem is that the theory diverges for the quantum vacuum, due to Lorentz invariance which imposes a spectral energy density for the vacuum fluctuations proportional to $\omega^{3}$ [2], the reason Ref. [1] introduced the cutoff frequency $\omega_{c}$ in Eq. (1). Third, stationary, homogeneous fields only impose $d(\rho \mathbf{v}) / d t=0$ [5] whereas the momentum itself is determined by the ME history. Fourth, to come to concrete predictions in realizable experiments one wishes to understand inhomogeneous media. The method employed in Ref. [1], based on pseudomomentum conservation, cannot be generalized for time-dependent fields, nor for inhomogeneous media.
In this Letter we address all four problems. To cope with the diverging vacuum without breaking Lorentz invariance we shall apply field regularization techniques [14]. To describe external fields that can be slowly switched on we shall consider total momentum balance, which is also free from the Abraham-Minkowski controversy [15]. The simplest inhomogeneous situation that can be regularized while respecting Lorentz invariance is the Casimir geometry [16], for which we shall make a precise prediction that is quite different from the FH. An important restriction of our work is the use of the macroscopic Maxwell equations, assuming that vacuum fluctuations are governed by the "macroscopic" properties of matter. Effects of dispersion and absorption caused by microscopic resonances cannot be described.

The optics of ME media is described by the constitutive relations of a bi-anisotropic form [3]

$$
\mathbf{D}=\varepsilon \cdot \mathbf{E}+\chi \cdot \mathbf{B} \quad \mathbf{H}=-\chi^{T} \cdot \mathbf{E}+\mu^{-1} \cdot \mathbf{B} .
$$

The tensors $\varepsilon$ and $\mu$ are assumed real valued and symmetric, the ME tensor $\chi$ only real valued, all frequency independent. All can be spatially varying and time dependent, but no dispersion or optical absorption is considered. In bianisotropic media, momentum conservation is expressed by,

$$
\partial_{t}\left(\frac{1}{4 \pi} \mathbf{E} \times \mathbf{B}+\rho \mathbf{v}\right)=\nabla \cdot\left(-\rho \mathbf{v v}+\mathcal{T}_{0}\right) .
$$

It features the symmetric stress tensor $\mathcal{T}_{0}$, with tensor elements $\quad 4 \pi \mathcal{T}_{0, i j}=\left(E_{i} E_{j}+B_{i} B_{j}\right)-\frac{1}{2}(\mathbf{E} \cdot \mathbf{E}+\mathbf{B}$. B) $\delta_{i j}$. Contrary to pseudomomentum, the "momentum" $\int d \mathbf{r}(\mathbf{E} \times \mathbf{B} / 4 \pi+\rho \mathbf{v})$ is conserved in the presence of sources, spatial inhomogeneities, or for a time dependence of the material parameters.

We shall discuss Lorentz invariance, and apply it only up to orders $v\left(c_{0}=1\right)$, which actually refers to Galilean invariance, but with the potential to be generalizable to Lorentz invariance. To describe ME effects we consider the 
Lagrangian density $\mathcal{L}(\mathbf{E}, \mathbf{B}, \mathbf{v})=-\rho \sqrt{1-\mathbf{v}^{2}}+\frac{1}{2}\left(\varepsilon \mathbf{E}^{2}-\right.$ $\left.\mathbf{B}^{2}\right)-(\varepsilon-1) \mathbf{E} \cdot(\mathbf{v} \times \mathbf{B})+\frac{\lambda}{2}\left(\mathbf{E}^{2}-\mathbf{B}^{2}\right)^{2}+\frac{\nu}{2}(\mathbf{E} \cdot \mathbf{B})^{2}$. If $\varepsilon=1$ it is Lorentz invariant since the last two terms are Lorentz scalars. The third term describes the Fizeau effect, known for more than a century, and corrects for the Galilean variance of the second term when $\varepsilon \neq 1$. The fields $\mathbf{D}, \mathbf{H}$ follow from the Euler-Lagrange formalism [4]. If we separate the dynamic wave fields $\mathbf{E}, \mathbf{B}$ from the static fields $\mathbf{E}_{0}, \mathbf{B}_{0}$, and linearize in the latter, we obtain the constitutive equations

$$
\begin{aligned}
\varepsilon_{i j}= & \varepsilon \delta_{i j}+2 \lambda\left(\mathbf{E}_{0}^{2}-\mathbf{B}_{0}^{2}\right) \delta_{i j}+4 \lambda E_{0, i} E_{0, j}+\nu B_{0, i} B_{0, j} \\
\mu_{i j}^{-1}= & \delta_{i j}+2 \lambda\left(\mathbf{E}_{0}^{2}-\mathbf{B}_{0}^{2}\right) \delta_{i j}-4 \lambda B_{0, i} B_{0, j}-\nu E_{0, i} E_{0, j} \\
\chi_{i j}= & (\varepsilon-1) \epsilon_{i j k} \boldsymbol{v}_{k}-4 \lambda E_{0, i} B_{0, j}+\nu\left(\mathbf{E}_{0} \cdot \mathbf{B}_{0}\right) \delta_{i j} \\
& +\nu B_{0, i} E_{0, j}
\end{aligned}
$$

where $\epsilon_{i j k}$ is the Lévi-Civita tensor. The Galilean transformation imposes that $\mathbf{E}^{\prime}=\mathbf{E}+\mathbf{v} \times \mathbf{B}$ and $\mathbf{B}^{\prime}=\mathbf{B}-$ $\mathbf{v} \times \mathbf{E}$ both for the static and the dynamic fields. Using Eq. (4) we can check that the macroscopic fields $\mathbf{D}$ and $\mathbf{H}$ defined by Eq. (2) transform to $\mathbf{D}^{\prime}=\mathbf{D}+\mathbf{v} \times \mathbf{H}$ and $\mathbf{H}^{\prime}=$ $\mathbf{H}-\mathbf{v} \times \mathbf{D}$, as they should in view of their interpretation as macroscopic electromagnetic fields. We conclude that Eqs. (2) and (4) provide a Galilean-invariant description of ME effects, with the potential to be generalizable to full Lorentz invariance.

The Galilean invariance of the FH now follows. For simplicity we adopt the geometry $\mathbf{E}_{0} \perp \mathbf{B}_{0}$ and $\mathbf{v} \| \mathbf{E}_{0} \times$ $\mathbf{B}_{0}$. We choose $\mathbf{E}_{0} \| \hat{\mathbf{z}}$ and $\mathbf{B}_{0} \| \hat{\mathbf{x}}$. With this choice, the $\chi$ tensor for a medium moving with velocity $\mathbf{v}$ takes the form

$$
\begin{aligned}
\chi= & (\varepsilon-1) v(\hat{\mathbf{z}} \hat{\mathbf{x}}-\hat{\mathbf{x}} \hat{\mathbf{z}}) \\
& +4 \pi(-4 \lambda \hat{\mathbf{z}} \hat{\mathbf{x}}+\nu \hat{\mathbf{x}} \hat{\mathbf{z}})\left(S_{0}+2 v \mathcal{E}_{0}\right),
\end{aligned}
$$

where the static fields are measured in the reference frame with respect to which the medium moves with speed $\mathbf{v}, \mathcal{E}_{0}$ is the electromagnetic energy density associated with the static fields, and $\mathbf{S}_{0}=(4 \pi)^{-1} \mathbf{E}_{0} \times \mathbf{B}_{0}$. The zero-point expectation value for the radiation momentum can be shown to be $\langle 0|\mathbf{E} \times \mathbf{B}| 0\rangle \propto \hbar K\left[(\lambda+4 \nu)\left(\mathbf{S}_{0}+2 \mathbf{v} \mathcal{E}_{0}\right)+\right.$ $(\varepsilon-1) \mathbf{v}]$ with $K=(2 \pi)^{-3} \int d^{3} \mathbf{k} \omega_{\mathbf{k}}$. Let us turn on adiabatically the external fields at $t=0$ with the medium at an initial velocity $\mathbf{v}_{0}$ with respect to the reference frame. Time integration of Eq. (3) gives

$$
\begin{aligned}
\rho \mathbf{v}-\rho \mathbf{v}_{0}+\hbar K(\lambda+4 \nu) & \left(\mathbf{S}_{0}+2 \mathbf{v} \mathcal{E}_{0}\right) \\
& +\hbar K(\varepsilon-1)\left(\mathbf{v}-\mathbf{v}_{0}\right)=0 .
\end{aligned}
$$

This equation is Galilean invariant, provided that $K$ is a genuine scalar. Indeed, being proportional to the total energy density of the quantum vacuum $K$ is rigourously Lorentz invariant [2], but diverging. Any physically acceptable regularization of $K$ must be Lorentz invariant, but this is not respected by cutting the integral off. This problem has been well studied for the Casimir effect, the attractive force between two parallel metallic plates, caused by the modified quantum vacuum. It is known to be insensitive to the vacuum energy density divergence, which has encouraged theoreticians to find a rigorous regularization of the vacuum energy density. The so-called dimensional regularization has been proposed [14], and gives acceptable results in all known cases. It consists of continuing the relation

$$
\int \frac{d^{d} \mathbf{k}}{(2 \pi)^{d}}\left(x^{2}+k^{2}\right)^{-p / 2} \equiv I_{x}(d, p)=\frac{x^{d-p}}{(4 \pi)^{d}} \frac{\Gamma\left(\frac{p-d}{2}\right)}{\Gamma\left(\frac{p}{2}\right)}
$$

beyond its strict domain of validity $p>d$. This regularizes $K \propto I_{0}(3,-1)=0$. So from dimensional regularization Eq. (6) predicts that $\mathbf{v}=\mathbf{v}_{0}$, and the conclusion is that no momentum is transferred from vacuum to an infinite homogeneous medium.

The final question we shall address is whether the FH occurs in an inhomogeneous medium. To this end we investigate the FH in the Casimir geometry (Fig. 1) and calculate $K$ as a function of the plate distance. We recall that Eq. (3) is valid when the constitutive tensors vary in space or time. We can thus think of changing the distance between the metallic plates, or switching on the fields $\mathbf{E}_{0}$, $\mathbf{B}_{0}$ slowly, with a ME slab with thickness $d$ initially at rest between the plates. Does the slab start moving as we turn on the fields or move the plates? The weakness of ME effects justifies the application of perturbation theory to include ME effects. If $\delta P$ is a small perturbation of the Hermitian operator $P$ - here the one associated with the Helmholtz equation - with real-valued eigenvalues $\omega_{n}^{2}$ and a complete set of orthonormal eigenfunctions $\left|\mathbf{E}_{n}\right\rangle$, the first-order change in eigenvalue is $\delta \omega_{n}^{2}=\left\langle\mathbf{E}_{n}|\delta P| \mathbf{E}_{n}\right\rangle$ [17]. The electromagnetic eigenmodes of the unperturbed Casimir geometry can be separated into TE and TM modes. The metallic boundary conditions impose that the tangential electric field vanishes on the plates $(z=0, L)$ as well as the normal magnetic field. Except for a factor $\exp (i \mathbf{k} \cdot \mathbf{x})$ the TE and TM modes are, respectively,

$$
\begin{aligned}
& \mathbf{E}_{n \mathbf{k}, T E}(z, \mathbf{x})=\sqrt{\frac{2}{L} \hat{\mathbf{k}} \times \hat{\mathbf{z}} \sin k_{n} z} \\
& \mathbf{B}_{n \mathbf{k}, T E}(z, \mathbf{x})=\sqrt{\frac{2}{L}}\left(\frac{k}{\omega} \hat{\mathbf{z}} \sin k_{n} z+i \frac{k_{n}}{\omega} \hat{\mathbf{k}} \cos k_{n} z\right)
\end{aligned}
$$

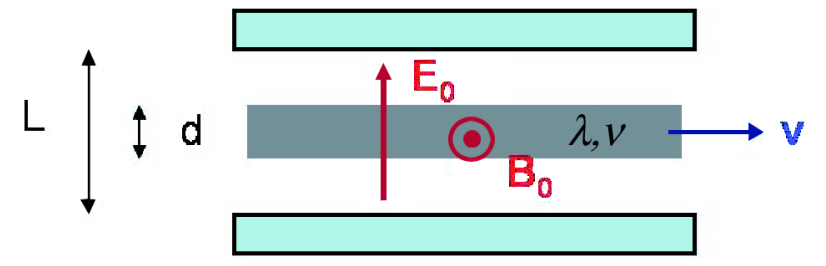

FIG. 1 (color online). The geometry considered to observe the Feigel process. The slab in the middle is subject to an electric field $\mathbf{E}_{0}$ and a magnetic field $\mathbf{B}_{0}$. As the Casimir plates approach each other, the slab starts moving with velocity $\mathbf{v}$. 


$$
\begin{aligned}
& \mathbf{E}_{n \mathbf{k}, T M}(z, \mathbf{x})=\sqrt{\frac{2}{L}}\left(\frac{k}{\omega} \hat{\mathbf{z}} \cos k_{n} z-i \frac{k_{n}}{\omega} \hat{\mathbf{k}} \sin k_{n} z\right) \\
& \mathbf{B}_{n \mathbf{k}, T M}(z, \mathbf{x})=-\sqrt{\frac{2}{L}} \hat{\mathbf{k}} \times \hat{\mathbf{z}} \cos k_{n} z
\end{aligned}
$$

with $\omega=\omega_{n \mathbf{k}} \equiv \sqrt{k^{2}+k_{n}^{2}}$ and $k_{n}=n \pi / L, n=1,2, \ldots$ for TE and $n=0,1, \ldots$ for TM. We will assume that the ME slab exhibits only ME effects, and put $\varepsilon=\mu=1$. The perturbation operator is then obtained from the Helmholtz equation, giving

$$
\delta P=\omega(\boldsymbol{\epsilon} \cdot \mathbf{p}) \cdot \chi^{T}(z)-\omega \chi(z) \cdot(\boldsymbol{\epsilon} \cdot \mathbf{p})
$$

with $\chi(z)=-4 \lambda \mathbf{E}_{0} \mathbf{B}_{0}+\nu\left(\mathbf{E}_{0} \cdot \mathbf{B}_{0}\right) I+\nu \mathbf{B}_{0} \mathbf{E}_{0}$ ( $I$ is the unit tensor) inside the ME slab and 0 outside. This ignores the contribution of $v \mathcal{E}_{0}$ to $\chi$ in Eq. (5) which is necessary to obey Galilean invariance, but which is in reality very small for the speed that we finally obtain. This leads to

$$
\begin{aligned}
\delta \omega_{n \mathbf{k}}^{2}(T E)= & -4 \omega k\left[4 \lambda \mathbf{E}_{0} \cdot(\hat{\mathbf{k}} \times \hat{\mathbf{z}})\left(\mathbf{B}_{0} \cdot \hat{\mathbf{z}}\right)\right. \\
& \left.-\nu \mathbf{B}_{0} \cdot(\hat{\mathbf{k}} \times \hat{\mathbf{z}})\left(\mathbf{E}_{0} \cdot \hat{\mathbf{z}}\right)\right] \times I_{n}^{s} \\
\delta \omega_{n \mathbf{k}}^{2}(T M)= & -4 \omega k\left[4 \lambda \mathbf{B}_{0} \cdot(\hat{\mathbf{k}} \times \hat{\mathbf{z}})\left(\mathbf{E}_{0} \cdot \hat{\mathbf{z}}\right)\right. \\
& \left.-\nu \mathbf{E}_{0} \cdot(\hat{\mathbf{k}} \times \hat{\mathbf{z}})\left(\mathbf{B}_{0} \cdot \hat{\mathbf{z}}\right)\right] \times I_{n}^{c}
\end{aligned}
$$

with the notation $I_{n}^{s}=L^{-1} \int_{L / 2-d / 2}^{L / 2+d / 2} d z \sin ^{2}\left(k_{n} z\right)$ and $I_{n}^{c}=$ $L^{-1} \int_{L / 2-d / 2}^{L / 2+d / 2} d z \cos ^{2}\left(k_{n} z\right)$.

We need to calculate the vacuum expectation value for $\mathbf{E}^{*} \times \mathbf{B}$. The integral over $z$ of $\left(\mathbf{E}^{*} \times \mathbf{B}\right) / 4 \pi$ gives the momentum density of radiation per unit area, which we shall denote by the vector $\mathbf{g}$. Use of Maxwell's equations leads to $\left(\mathbf{E}^{*} \times \mathbf{B}\right)(\omega)_{i}=-\epsilon_{i j k} E_{j}^{*}(\omega) \times$ $\omega^{-1}(\boldsymbol{\epsilon} \cdot \mathbf{p})_{k l} E_{l}(\omega)$. Since $\epsilon_{i j k}(\boldsymbol{\epsilon} \cdot \mathbf{p})_{k l}=\delta_{i l} p_{j}-\delta_{j l} p_{i}$ we get as an intermediate step $\left\langle 0\left|\left(\mathbf{E}^{*} \times \mathbf{B}\right)(\omega)_{i}\right| 0\right\rangle=$ $\omega^{-1} \sum_{j}\left\langle 0\left|E_{j}^{*}(\omega) p_{i} E_{j}(\omega)-E_{j}^{*}(\omega) p_{j} E_{i}(\omega)\right| 0\right\rangle$. The quantum vacuum expectation for the product of two electric fields is supplied by the fluctuation-dissipation theorem, that we shall apply here for zero temperature, valid when $T<\hbar c_{0} / k L=500 \mathrm{~K} / L[\mu \mathrm{m}]$ [18]. Let $G$ be the Green's tensor associated with the Helmholtz equation for $\mathbf{E}$

$$
G(\omega, \mathbf{p})=\left[\omega^{2}+\omega(\epsilon \cdot \mathbf{p}) \cdot \chi^{T}-\omega \chi \cdot(\epsilon \cdot \mathbf{p})+(\epsilon \cdot \mathbf{p})^{2}\right]^{-1} .
$$

Application of $\left\langle 0\left|E_{i}(\omega, \mathbf{r}) E_{j}^{*}\left(\omega, \mathbf{r}^{\prime}\right)\right| 0\right\rangle=-2 \hbar \omega^{2}\langle\mathbf{r}| \times$ $\operatorname{Im} G_{i j}(\omega, \mathbf{p})\left|\mathbf{r}^{\prime}\right\rangle[19]$ gives us

$$
\frac{1}{4 \pi}\left\langle 0\left|\left(\mathbf{E}^{*} \times \mathbf{B}\right)_{i}(\mathbf{r})\right| 0\right\rangle=-\frac{1}{2 \pi} \hbar \int_{0}^{\infty} \frac{d \omega}{2 \pi} \omega \cdot \sum_{j}\left\{p_{i} \operatorname{Im} G_{j j}(\omega, \mathbf{r})-p_{j} \operatorname{Im} G_{i j}(\omega, \mathbf{r})\right\},
$$

where we recall that $\mathbf{p}=-i \nabla=\mathbf{k}-i \hat{\mathbf{z}} \partial_{z}$. We insert the spectral decomposition of the Green's tensor,

$$
-\operatorname{Im} G_{i j}\left(\mathbf{x}, z, \mathbf{x}^{\prime}, z^{\prime}, \omega\right)=\sum_{n} \int \frac{d^{2} \mathbf{k}}{(2 \pi)^{2}} E_{i}^{(n \mathbf{k})}(z) E_{j}^{*(n \mathbf{k})}(z) \pi \delta\left(\omega^{2}-\omega_{n \mathbf{k}}^{2}\right) \mathrm{e}^{i \mathbf{k} \cdot\left(\mathbf{x}-\mathbf{x}^{\prime}\right)}
$$

Since the eigenfunctions are normalized, the $z$ integral of the first term of Eq. (14) equals $\mathbf{k} \delta\left(\omega^{2}-\omega_{n \mathbf{k}}^{2}\right)$ and can thus be conveniently expressed in terms of the eigenvalues only. This is not the case for the second term, but we can apply the Maxwell relation $\nabla \cdot \mathbf{D}=0$ to find that $\mathbf{p} \cdot \mathbf{E}=$ $-\mathbf{p} \cdot \chi \cdot \mathbf{B}$. Since this is already proportional to $\chi$, we can use the unperturbed eigenfunctions and eigenvalues. We can rearrange the result for the radiative momentum density per unit area to

$$
\begin{aligned}
\langle 0|\mathbf{g}| 0\rangle= & \frac{1}{2} \hbar \int_{0}^{\infty} \frac{d \omega}{2 \pi} \omega \sum_{n} \int \frac{d^{2} \mathbf{k}}{(2 \pi)^{2}} \mathbf{k} \delta\left(\omega^{2}-\omega_{n \mathbf{k}}^{2}\right) \\
& -\frac{\hbar}{8 \pi i} \sum_{n} \int \frac{d^{2} \mathbf{k}}{(2 \pi)^{2}} \\
& \times \int_{0}^{L} d z E_{i}^{(n \mathbf{k})}(z) \partial_{j} \chi_{j l} B_{l}^{*,(n \mathbf{k})}(z) .
\end{aligned}
$$

We shall see that both the summation over the modes and the integral over horizontal wave number diverge. Apart from the dimensional regularization of Eq. (7) we shall apply here the so-called zeta function regularization of the discrete sum, $\sum_{n=0}^{\infty} n^{-s}=\zeta(s)$ which is continued analytically to $s \leq 1$ [20]. In particular, $\sum_{n=0}^{\infty} n^{3} \rightarrow$ $\zeta(-3)=1 / 120$. The regularization of oscillatory terms is performed by introducing $\epsilon>0$,

$$
\sum_{n=0}^{\infty} \mathrm{e}^{-n \epsilon} \sin n \theta=\frac{1}{2} \frac{\sin \theta}{\cosh \epsilon-\cos \theta}
$$

For $\theta=0, \pi$ this sum equals zero if we take the limit $\epsilon \downarrow 0$ afterwards. If $\theta \neq 0, \pi$ the sum gives $\cos (\theta / 2) / 2 \sin (\theta / 2)$. For future need we give the following regularization that follows from Eq. (17),

$$
\sum_{n=0}^{\infty} k_{n}^{3} \sin ^{2} k_{n} z \rightarrow \frac{\pi^{3}}{240 L^{3}}\left(1+\frac{15 L}{\pi} \frac{\partial}{\partial z} \frac{\cos \pi z / L}{\sin ^{3} \pi z / L}\right)
$$

The first term of Eq. (16) can be evaluated using Eqs. (11) and (12): $\omega_{n \mathbf{k}}^{2}(\mathrm{TE}) \approx k_{n}^{2}+\left(\mathbf{k}+8 \pi \omega \nu I_{n}^{s} \mathbf{S}_{0}\right)^{2}$ and $\omega_{n \mathbf{k}}^{2}(\mathrm{TM}) \approx k_{n}^{2}+\left(\mathbf{k}+32 \pi \omega \lambda I_{n}^{c} \mathbf{S}_{0}\right)^{2}$. Upon an appropriate change of base in the $\mathbf{k}$ integral we obtain 


$$
\begin{gathered}
\mathbf{g}_{1}(\mathrm{TE})=-\hbar \nu \mathbf{S}_{0} \sum_{n} \int \frac{d^{2} \mathbf{k}}{(2 \pi)^{2}} \omega_{n \mathbf{k}} I_{n}^{s} \\
\mathbf{g}_{1}(\mathrm{TM})=-4 \hbar \lambda \mathbf{S}_{0} \sum_{n} \int \frac{d^{2} \mathbf{k}}{(2 \pi)^{2}} \omega_{n \mathbf{k}} I_{n}^{c} .
\end{gathered}
$$

With the proposed schemes we can regularize Eqs. (18) and (19) resulting in

$$
\mathbf{g}_{1}=\frac{\pi^{2}}{1440} \frac{\hbar d}{L^{4}}\left[(4 \lambda+\nu)+(4 \lambda-\nu) \frac{30 L}{\pi d} \frac{\sin \pi d / 2 L}{\cos ^{3} \pi d / 2 L}\right] \mathbf{S}_{0} .
$$

The second term of Eq. (16) can be handled similarly, eliminating the discontinuity of $\lambda$ and $\nu$ at the ME slab boundaries using one integration by parts. Dimensional regularization gives $(2 \pi)^{-2} \int d^{2} \mathbf{k} \omega_{n \mathbf{k}} \rightarrow-k_{n}^{3} / 6 \pi$ and $(2 \pi)^{-2} \int d^{2} \mathbf{k} k_{n}^{2} / \omega_{n \mathbf{k}} \rightarrow-k_{n}^{3} / 2 \pi$. The result is

$$
\mathbf{g}_{2}=\frac{\pi^{2}}{1440} \frac{\hbar d}{L^{4}}\left[(\nu-12 \lambda)-(\nu+12 \lambda) \frac{30 L}{\pi d} \frac{\sin \pi d / 2 L}{\cos ^{3} \pi d / 2 L}\right] \mathbf{S}_{0}
$$

The final regularized result for the total radiation momentum of the zero-point fluctuations per unit area is the sum of (20) and (21),

$$
\mathbf{g}=\frac{\pi^{2} \hbar d}{720 L^{4}}\left[(\nu-4 \lambda)-(\nu+4 \lambda) \frac{30 L}{\pi d} \frac{\sin (\pi d / 2 L)}{\cos ^{3}(\pi d / 2 L)}\right] \mathbf{S}_{0}
$$

For $0 \leq d<L$ it is found that the result is dominated by the antisymmetric part of the ME tensor $\chi$, proportional to $\nu+4 \lambda$. Hence we can identify $K(L, d) \approx-\left(\pi / 24 d L^{3}\right) \times$ $\sin (\pi d / 2 L) / \cos ^{3}(\pi d / 2 L)$, and apply Eq. (6) to describe the FH. Upon comparing to the original FH in Eq. (1) one identifies a frequency cutoff of the order $\pi c_{0} / L$ which is much smaller than the typical frequency beyond which $\mathrm{ME}$ response is believed to disappear and validates our neglect of resonances and absorption.

To observe the FH we can imagine the plates to be largely separated $(L=\infty)$, and the ME slab at rest with respect to these plates $[\mathbf{v}(0)=0]$, with the fields $\mathbf{E}_{0}$ and $\mathbf{B}_{0}$ switched on. We can now let the plates approach with opposite momentum until a finite distance L. Actually, the zero-point fluctuations will do this for us by means of the Casimir effect [21]. This converts vacuum energy into kinetic energy, but no momentum is put into the system, since the plates always have opposite momentum. Yet, the radiation momentum density $\mathbf{g}$ per unit area of the squeezed quantum vacuum changes with $L$, and must be compensated by the matter momentum density per unit area $\rho d \mathbf{v}$. We conclude that the medium acquires a momentum density $-\mathbf{g}$ per unit area from the vacuum fluctuations, with $\mathbf{g}$ given by (22). Assuming that the experimental values for magneto-electric birefringence in molecular liquids $\left(\rho \approx 2000 \mathrm{~kg} / \mathrm{m}^{3}\right)$ [10] are frequency independent, and taking $d=L / 2$ and $L=1 \mu \mathrm{m}$, Eq. (22) predicts $\rho v=4 \times 10^{-23} \mathrm{~g} \mathrm{~cm}^{-2} \mathrm{~s}^{-1} \quad\left(v=10^{-17} \mathrm{~nm} / \mathrm{s}\right)$ for such media. The $\mathrm{FH}$ is thus valid for the standard Casimir geometry, but it is immeasurably small. The prediction of Eq. (1), however, $v=50 \mathrm{~nm} / \mathrm{s}$ would be within experimental reach. Since, contrary to the Casimir effect, the FH is explicitly sensitive to regularization, its experimental falsification would support dimensional regularization of vacuum energy.

In conclusion, we have formulated a regularized, Galilean-invariant field theory for the transfer of momentum from vacuum to magneto-electric matter. We conclude that no transfer occurs in infinite media. A small momentum transfer happens in a squeezed vacuum. This opens up the possibility for future work to study momentum transfer from vacuum to other objects such as a small Mie sphere, where the effect might be enhanced near geometric eigenfrequencies, and whose motion might be manipulated by vacuum fluctuations (a "zero-point tweezer").

This work was supported by the Advanced Concept Team of the European Space Agency, Ariadne call 18806/04/NL/MV. We would like to thank N. Lan and A. Rathke for comments and discussions, and A. Feigel for his comments.

[1] A. Feigel, Phys. Rev. Lett. 92, 020404 (2004); In this work $\Delta n$ stands for the component of $\chi \mathbf{E}_{0} \times \mathbf{B}_{0}$ along the direction of momentum in Eq. (1).

[2] P.W. Miloni, The Quantum Vacuum (Academic, New York, 1994).

[3] Th. O'Dell, The Electrodynamics of Magneto-electric Media (North-Holland, Amsterdam, 1970).

[4] G. L. J. A. Rikken and C. Rizzo, Phys. Rev. A 67, 015801 (2003).

[5] B. A. van Tiggelen and G. L. J. A. Rikken, Phys. Rev. Lett. 93, 268903 (2004).

[6] R. Schützhold and G. Plunien, Phys. Rev. Lett. 93, 268901 (2004).

[7] A. Feigel, Phys. Rev. Lett. 93, 268904 (2004).

[8] G. T. Rado, Phys. Rev. Lett. 13, 335 (1964).

[9] R. Pisarev et al., Phase Transit. 37, 63 (1991).

[10] T. Roth and G. L. J. A. Rikken, Phys. Rev. Lett. 88, 063001 (2002).

[11] J. H. Jung et al., Phys. Rev. Lett. 93, 037403 (2004).

[12] M. Kubota et al., Phys. Rev. Lett. 92, 137401 (2004).

[13] G.L. J. A. Rikken, C. Strohm, and P. Wyder, Phys. Rev. Lett. 89, 133005 (2002).

[14] X. Kong and F. Ravndal, Phys. Rev. Lett. 79, 545 (1997).

[15] I. Brevik, Phys. Rep. 52, 133 (1979). For a recent discussion, see: R. Loudon, Fortschr. Phys. 52, 1134 (2004).

[16] H. B. G. Casimir, Proc. K. Ned. Akad. Wet. 51, 793 (1948).

[17] E. Merzbaccher, Quantum Mechanics (Wiley, New York, 1970).

[18] L. S. Brown and G. J. Maclay, Phys. Rev. 184, 1272 (1969).

[19] L.D. Landau and E.M. Lifschitz, Statistical Physics (Pergamon, New York, 1980), Part 1.

[20] E. Elizalde, J. Phys. A 27, L299 (1994).

[21] M. J. Sparnaay, Physica (Amsterdam) 24, 751 (1958); S. K. Lamoreaux, Phys. Rev. Lett. 78, 5 (1997). 